\title{
FINITE ELEMENT APPROXIMATION FOR THE FRACTIONAL EIGENVALUE PROBLEM
}

\author{
JUAN PABLO BORTHAGARAY, LEANDRO M. DEL PEZZO, AND SANDRA MARTÍNEZ
}

\begin{abstract}
The purpose of this work is to study a finite element method for finding solutions to the eigenvalue problem for the fractional Laplacian. We prove that the discrete eigenvalue problem converges to the continuous one and we show the order of such convergence. Finally, we perform some numerical experiments and compare our results with previous work by other authors.
\end{abstract}

\section{Introduction and Main Results}

Anomalous diffusion phenomena are ubiquitous in nature [33, 42, and the study of nonlocal operators has been an active area of research in different branches of mathematics. Such operators arise in applications as image processing [13, 26, 28, 39], finance [15, 18, electromagnetic fluids [41, peridynamics [53], porous media flow [8, 19, among others.

One striking example of a nonlocal operator is the fractional Laplacian $(-\Delta)^{s}$, defined by

$$
(-\Delta)^{s} u(x):=2 C(n, s) \int_{\mathbb{R}^{n}} \frac{u(x)-u(y)}{|x-y|^{n+2 s}} d y, \quad x \in \mathbb{R}^{n} .
$$

Here the integral is understood in the principal value sense and the normalization constant $C(n, s)$ is given by

$$
C(n, s):=\frac{2^{2 s-1} s \Gamma\left(s+\frac{n}{2}\right)}{\pi^{n / 2} \Gamma(1-s)} .
$$

In the theory of stochastic processes, this operator appears as the infinitesimal generator of a stable Lévy process, see for instance [9, 55]. Moreover, the fractional Laplacian is also one of the simplest examples of a pseudo-differential operator, because its symbol is just $P(\xi)=|\xi|^{2 s}$.

An interesting problem concerning the fractional Laplacian is to find its eigenspaces on bounded domains. Namely, to find a positive number $\lambda$ (eigenvalue) and a function $u \neq \equiv 0$ (eigenfunction) such that

$$
\left\{\begin{aligned}
(-\Delta)^{s} u=\lambda u & \text { in } \Omega, \\
u=0 & \text { in } \Omega^{c}=\mathbb{R}^{n} \backslash \Omega,
\end{aligned}\right.
$$

2010 Mathematics Subject Classification. 46E35, 35P15, 49R05 65N25, 65N30.

Key words and phrases. Fractional Laplacian, eigenvalue problem, finite element method.

Research of the first author has been partially supported by CONICET under grant PIP 2014$201611220130100184 \mathrm{CO}$ 
where $\Omega$ is a bounded domain in $\mathbb{R}^{n}$ and $s \in(0,1)$. Observe that, due to the fact that pointwise values of $(-\Delta)^{s} u$ depend on the value of $u$ over the whole space, boundary conditions need to be substituted by volume constraints on the complement of $\Omega$.

A natural application of the problem we are considering in this paper is given by the fractional Schrödinger equation. This equation arises from extending the Feynman path integral approach from Brownian-like quantum mecanical paths that lead to the classical Schrödinger equation- to Lévy-like paths [37]. In this regard, eigenfunctions of the fractional Laplacian correspond to the energy states of the system being modeled. This has motivated researchers to study this problem, both from the physical, mathematical and computational point of view. Among the various references in these subjects, we refer the reader to [4, 15, 7, 16, 23, 40] for further details.

Even if $\Omega$ is an interval, it is very challenging to obtain closed analytical expressions for the eigenvalues and eigenfunctions of the fractional Laplacian. This motivates the utilization of discrete approximations of this problem (see, for example, [27, 36, 57]); in this work we consider a finite element method. In first place, we prove that the discrete eigenvalue problem converges to the continuous one. Then, we show the order of convergence for eigenvalues and eigenfunctions, both in the energy and the $L^{2}$-norm. Orders of convergence are increased by considering suitably graded meshes that stem from a precise characterization of the behavior of eigenfunctions near the boundary of $\Omega$. Finally, we perform some numerical experiments and compare our results with previous work by other authors. These results are in good agreement with our theory.

The finite element method is flexible enough to deal with non-convex domains, and enables us to provide estimates and sharp upper bounds for eigenvalues even in this context. Moreover, as a consequence of our numerical experiments in the $L$-shaped domain $\Omega=[-1,1]^{2} \backslash[0,1]^{2}$, we conjecture that the first eigenfunction for this domain is as regular as the first one in any smooth domain.

Due to the nonlocal nature of the problem, a straightforward implementation of the finite element method demands a double loop over the elements to assembly the stiffness matrix. Thus, the complexity of this routine is quadratic with respect to the number of elements. As reported in [1], this step requires about $99 \%$ of the total CPU time. This issue has been tackled in [3], where sparse approximations of the stiffness matrix have been proposed and analyzed. Furthermore, the condition number of the stiffness matrix $A(\kappa(A))$ corresponding to the fractional Laplacian of order $s$ using standard piecewise linear finite elements over a mesh with size $h$ scales as $\kappa(A) \simeq h^{-2 s}$. Therefore, in the numerical examples performed for this work we have solved the discrete systems by using a direct solver.

Main Results. In order to state our results we need to collect some notation and definitions. The natural functional space for the eigenvalue problem $[1.1$ is

$$
\widetilde{H}^{s}(\Omega):=\left\{v \in H^{s}\left(\mathbb{R}^{n}\right): \operatorname{supp} v \subset \bar{\Omega}\right\},
$$

where $H^{s}\left(\mathbb{R}^{n}\right)$ is the space of all functions $u \in L^{2}\left(\mathbb{R}^{n}\right)$ such that

$$
|v|_{H^{s}\left(\mathbb{R}^{n}\right)}^{2}:=\iint_{\mathbb{R}^{2 n}} \frac{|v(x)-v(y)|^{2}}{|x-y|^{n+2 s}} d x d y<\infty .
$$


Moreover, $\left(\mathbb{V},\|\cdot\|_{\mathbb{V}}\right):=\left(\widetilde{H}^{s}(\Omega), \sqrt{C(n, s)}|\cdot|_{H^{s}\left(\mathbb{R}^{n}\right)}\right)$ is a Hilbert space with the inner product

$$
\langle u, v\rangle:=C(n, s) \iint_{\mathbb{R}^{2 n}} \frac{(u(x)-u(y))(v(x)-v(y))}{|x-y|^{n+2 s}} d x d y .
$$

Obviously, the constant $\sqrt{C(n, s)}$ has no effect on the definition of the space; it is included in order to make the notation simpler in the rest of the paper. The fractional space $H^{s}\left(\mathbb{R}^{n}\right)$ can also be defined for any $s>1$. If $s=m+\sigma$, where $m \in \mathbb{N}$ and $\sigma \in(0,1), H^{s}\left(\mathbb{R}^{n}\right)$ is the space of all functions $v \in H^{m}\left(\mathbb{R}^{m}\right)$ such that its weak derivatives of order $m$ belong to $H^{\sigma}\left(\mathbb{R}^{n}\right)$. For more details, see Section 2 .

In this context, the eigenvalue problem 1.1 has the following variational formulation: find $\lambda \in(0,+\infty)$ and $u \in \mathbb{V}$ such that $u \neq \equiv$ and

$$
\langle u, v\rangle=\lambda(u, v) \quad \text { for all } v \in \mathbb{V},
$$

where $(\cdot, \cdot): L^{2}(\Omega) \times L^{2}(\Omega) \rightarrow \mathbb{R}$ is the bilinear form

$$
(u, v):=\int_{\Omega} u(x) v(x) d x
$$

It is well-known (see, for example, [49]) that there is an infinite sequence of eigenvalues $\left\{\lambda^{(k)}\right\}_{k \in \mathbb{N}}$,

$$
0<\lambda^{(1)}<\lambda^{(2)} \leq \cdots \leq \lambda^{(k)} \leq \cdots, \quad \lambda^{(k)} \rightarrow \infty \text { as } k \rightarrow \infty,
$$

where the same eigenvalue can be repeated several times according to its multiplicity. The corresponding eigenfunctions $u^{(k)}$, normalized by $\left\|u^{(k)}\right\|_{L^{2}(\Omega)}=1$, form a complete orthonormal set in $L^{2}(\Omega)$. See also [47, 50, 52] and the references therein for further details on the fractional eigenvalue problem.

Let us now introduce the discrete space. Let $\mathcal{T}_{h}$ be a family of triangulations of $\Omega$ satisfying

(Regularity)

$$
\exists \sigma>0 \text { s.t. } h_{T} \leq \sigma \rho_{T},
$$

for any element $T \in \mathcal{T}_{h}$, where $h_{T}$ is the diameter of $T$ and $\rho_{T}$ is the radius of the largest ball contained in $T$. This is the only requirement we need to impose to our family of triangulations.

We consider continuous piecewise linear functions on $\mathcal{T}_{h}$, namely

$$
\mathbb{V}_{h}:=\left\{v \in \mathbb{V}:\left.v\right|_{T} \in \mathcal{P}_{1}(T) \forall T \in \mathcal{T}_{h}\right\}
$$

Our Galerkin approximation consists in looking for discrete eigenvalues $\lambda_{h} \in \mathbb{R}$ and $u_{h} \in \mathbb{V}_{h}$ such that $u_{h} \not \equiv 0$ and

$$
\left\langle u_{h}, v\right\rangle=\lambda_{h}\left(u_{h}, v\right) \quad \forall v \in \mathbb{V}_{h} .
$$

We can order the discrete eigenvalues of 1.3 as follows

$$
0<\lambda_{h}^{(1)} \leq \lambda_{h}^{(2)} \leq \cdots \leq \lambda_{h}^{(k)} \leq \cdots \leq \lambda_{h}^{\left(\operatorname{dim} \mathbb{V}_{h}\right)},
$$

where the same eigenvalue is repeated according to its multiplicity. The corresponding eigenfunctions $u_{h}^{(k)}$ (normalized by $\left\|u_{h}^{(k)}\right\|_{L^{2}(\Omega)}=1$ ) form an orthonormal set in $L^{2}(\Omega)$.

The purpose of this work is to prove the convergence of the discrete problem 1.3 to the continuous 1.2 . 
Our first result concerning the convergence is to determine it in gap distance, that is, we prove that the discrete eigenvalue problem converges to the continuous, following the definition of convergence given in [32, 11] (see also [10]). More precisely, we define the gap between Hilbert spaces $E, F \subset H$ by

$$
\delta(E, F)=\sup _{u \in E,\|u\|_{H}=1} \inf _{v \in F}\|u-v\|_{H}, \quad \hat{\delta}(E, F)=\max (\delta(E, F), \delta(F, E)) .
$$

Then, taking $H=\mathbb{V}$, we say that the discrete eigenvalue problem 1.3 converges to the continuous one 1.2 if, for any $\varepsilon>0$ and $k>0$, there is $h_{0}>0$ such that

$$
\max _{1 \leq i \leq m(k)}\left|\lambda^{(i)}-\lambda_{h}^{(i)}\right| \leq \varepsilon, \quad \hat{\delta}\left(\bigoplus_{i=1}^{m(k)} E^{(i)}, \bigoplus_{i=1}^{m(k)} E_{h}^{(i)}\right) \leq \varepsilon,
$$

for all $h<h_{0}$. Here, $m(k)$ is the dimension of the space spanned by the first distinct $k$ eigenspaces and $E^{(i)}$ and $E_{h}^{(i)}$ are the eigenspace and the discrete eigenspace associated to $\lambda^{(i)}$ and $\lambda_{h}^{(i)}$, respectively.

Remark 1.1. We remark here that to obtain convergence in gap distance, we only need to assume that the extension $H^{s}(\Omega) \rightarrow H^{s}\left(\mathbb{R}^{n}\right)$ is continuous. This is in turn equivalent to the following condition [56]: there is a constant $C>0$ such that for all $x \in \Omega$ and all $r \in(0,1]$,

$$
|\Omega \cap B(x, r)| \geq C r^{n} \quad \forall x \in \Omega .
$$

Theorem 1.2. If $\Omega$ is a fractional extension domain, then the discrete eigenvalue problem 1.3 converges to the continuous one 1.2 .

Having established the convergence of the discrete problem to the continuous, we next state the order of such a convergence. To this end, we need to provide a Sobolev regularity result. This, in turn, requires some additional assumptions on the domain. We prove the following.

Proposition 1.3. Let $\Omega \subset \mathbb{R}^{n}$ be a Lipschitz domain satisfying the exterior ball condition and let $u$ be an eigenfunction of $(-\Delta)^{s}$ in $\Omega$ with homogeneous Dirichlet boundary conditions. Then, $u \in \widetilde{H}^{s+1 / 2-\varepsilon}(\Omega)$ for any $\varepsilon>0$.

Moreover, considering the weighted Sobolev scale (cf. (2.3) below) it also holds that $u \in H_{1 / 2-2 \varepsilon}^{1+s-\varepsilon}\left(\mathbb{R}^{n}\right)$ for any $\varepsilon>0$.

The regularity in standard spaces in the previous proposition is utilized to prove an a priori error bound for the finite element approximations with meshes satisfying (Regularity). Moreover, the weighted regularity above enables to consider suitably graded meshes (see the definition $(\mathrm{H})$ in Subsection 2.4), and these deliver an enhanced order of convergence. Upon proving approximation properties of the discrete spaces considered, an application of Babuška-Osborn theory [6] allows to deduce the rate of convergence for the eigenvalues and for the eigenfunctions in the energy norm, both for uniform and graded meshes.

Theorem 1.4. Let $\Omega \subset \mathbb{R}^{n}$ be a Lipschitz domain satisfying the exterior ball condition and let $\lambda^{(k)}$ be an eigenvalue of multiplicity $m$ (that $i s, \lambda^{(k)}=\lambda^{(k+1)}=$ $\cdots=\lambda^{(k+m-1)}$ and $\lambda^{(i)} \neq \lambda^{(k)}$ for $\left.i \neq k, \ldots, k+m-1\right)$. Consider the Galerkin approximations given by 1.3 on a shape-regular familiy of meshes. 
FE APPROXIMATION FOR THE FRACTIONAL EIGENVALUE PROBLEM

(1) For any $\varepsilon>0$, there exists a positive constant $C$ independent of $h$ such that

$$
0 \leq \lambda_{h}^{(j)}-\lambda^{(k)} \leq C h^{1-\varepsilon} \quad \forall k \leq j \leq k+m-1 .
$$

Moreover, if $u^{(k)}$ is an eigenfunction associated to $\lambda^{(k)}$, there is

$$
\left\{w_{h}^{(k)}\right\} \subset E_{h}^{(k)} \oplus \ldots \oplus E_{h}^{(k+m-1)}
$$

such that

$$
\left\|u^{(k)}-w_{h}^{(k)}\right\|_{\mathbb{V}} \leq C h^{1 / 2-\varepsilon} .
$$

(2) On the other hand, if $s>1 / 2$ and the meshes are graded according to $(\mathrm{H})$ with $\mu=2$, then the estimates above can be refined to be

$$
0 \leq \lambda_{h}^{(j)}-\lambda^{(k)} \leq C h^{2-\varepsilon} \quad \forall k \leq j \leq k+m-1 .
$$

and

$$
\left\|u^{(k)}-w_{h}^{(k)}\right\|_{\mathbb{V}} \leq C h^{1-\varepsilon} .
$$

Finally, to prove convergence orders in the $L^{2}$ norm, we require smoothness on the domain. This regularity assumption is required in order to apply an AubinNitsche duality argument. We obtain the following.

Theorem 1.5. Assume $\Omega$ is a smooth domain and let $\alpha=\min \{s, 1 / 2-\varepsilon\}$ for any $\varepsilon>0$. Then, if $\lambda^{(k)}$ is an eigenvalue of multiplicity $m$ and if $u^{(k)}$ is an eigenfunction associated to $\lambda^{(k)}$, there is

$$
\left\{w_{h}^{(k)}\right\} \subset E_{h}^{(k)} \oplus \ldots \oplus E_{h}^{(k+m-1)}
$$

such that

$$
\left\|u^{(k)}-w_{h}^{(k)}\right\|_{L^{2}(\Omega)} \leq C h^{\alpha+1 / 2-\varepsilon} .
$$

In order to illustrate the convergence estimates obtained in Theorems 1.4 and 1.5. we present the results of numerical tests for finite element discretizations of one and two-dimensional eigenvalue problems. Moreover, in the latter case, some examples in domains that do not satisfy the hypotheses of Proposition 1.3 are displayed. These examples provide numerical evidence that the assertion of this proposition still holds true under weaker assumptions about the domain.

The paper is organized as follows. Section 2 collects the notation we employ, and reviews some previous works on the problem under consideration. In particular, regularity of eigenfunctions is proved. The section concludes with a discussion of certain aspects of finite element approximations of the fractional Laplacian. Afterwards, Section 3 deals with the convergence of the discrete eigenvalue problem to the continuous one in gap distance. In Section 4 , proof of the orders of convergence for eigenvalues and eigenfunctions are given, including estimates for graded meshes. Finally, numerical experiments are discussed in Section 5.

\section{Preliminaries and Definitions}

In this section we review the basic aspects of the problem under consideration. In first place, we set notation regarding Sobolev spaces. Afterwards, we analyze theoretical properties of the eigenvalue problem (1.1). Regularity results for weak solutions of fractional Laplace equations are recalled next. The section concludes with the introduction of the finite element spaces we work with. 
2.1. Sobolev spaces. Given an open set $\Omega \subset \mathbb{R}^{n}$ and $s \in(0,1)$, define the fractional Sobolev space $H^{s}(\Omega)$ as

$$
H^{s}(\Omega):=\left\{v \in L^{2}(\Omega):|v|_{H^{s}(\Omega)}<\infty\right\},
$$

where $|\cdot|_{H^{s}(\Omega)}$ is the seminorm

$$
|v|_{H^{s}(\Omega)}^{2}:=\iint_{\Omega^{2}} \frac{|v(x)-v(y)|^{2}}{|x-y|^{n+2 s}} d x d y .
$$

Obviously, $H^{s}(\Omega)$ is a Hilbert space endowed with the norm $\|\cdot\|_{H^{s}(\Omega)}=\|\cdot\|_{L^{2}(\Omega)}+$ $|\cdot|_{H^{s}(\Omega)}$. If $s>1$ and it is not an integer, the decomposition $s=m+\sigma$, where $m \in \mathbb{N}$ and $\sigma \in(0,1)$, allows to define $H^{s}(\Omega)$ by setting

$$
H^{s}(\Omega):=\left\{v \in H^{m}(\Omega):\left|D^{\alpha} v\right|_{H^{\sigma}(\Omega)}<\infty \text { for all } \alpha \text { s.t }|\alpha|=m\right\} .
$$

Let us also define the space of functions supported in $\Omega$,

$$
\widetilde{H}^{s}(\Omega):=\left\{v \in H^{s}\left(\mathbb{R}^{n}\right): \operatorname{supp} v \subset \bar{\Omega}\right\} .
$$

For $0 \leq s \leq 1$ and if $\Omega$ is a Lipschitz domain, this space may be defined through interpolation,

$$
\widetilde{H}^{s}(\Omega)=\left[L^{2}(\Omega), H_{0}^{1}(\Omega)\right]_{s} .
$$

Moreover, depending on the value of $s$, different characterizations of this space are available (see, for example [38, Chapter 11]). If $s<1 / 2$ the space $\widetilde{H}^{s}(\Omega)$ coincides with $H^{s}(\Omega)$, and if $s>1 / 2$ it may be characterized as the closure of $C_{0}^{\infty}(\Omega)$ with respect to the $|\cdot|_{H^{s}(\Omega)}$ norm. In the latter case, it is also customary to denote it by $H_{0}^{s}(\Omega)$. The particular case of $s=1 / 2$ gives raise to the Lions-Magenes space $H_{00}^{1 / 2}(\Omega)$, which can be characterized by

$$
H_{00}^{1 / 2}(\Omega):=\left\{v \in H^{1 / 2}(\Omega): \int_{\Omega} \frac{v(x)^{2}}{\operatorname{dist}(x, \partial \Omega)} d x<\infty\right\} .
$$

Note that the inclusion $H_{00}^{1 / 2}(\Omega) \subset H_{0}^{1 / 2}(\Omega)=H^{1 / 2}(\Omega)$ is strict.

It is apparent that $\langle\cdot, \cdot\rangle$ defines an inner product on $\widetilde{H}^{s}(\Omega)$. In addition, the norm induced by it, which is just the $H^{s}\left(\mathbb{R}^{n}\right)$ seminorm, is equivalent to the full $H^{s}\left(\mathbb{R}^{n}\right)$ norm on this space, because of the following well known result. See for instance [20, Lemma 2.5].

Proposition 2.1 (Poincaré inequality). Let $\Omega$ be a bounded domain, then there is a constant $c=c(\Omega, n, s)$ such that

$$
\|v\|_{L^{2}(\Omega)} \leq c|v|_{H^{s}\left(\mathbb{R}^{n}\right)} \quad \forall v \in \widetilde{H}^{s}(\Omega) .
$$

For the proof of the following result, see e.g. [21, 22].

Proposition 2.2. Let $\Omega$ be an extension domain (cf. Remark 1.1). Then, the inclusion $\widetilde{H}^{s}(\Omega) \hookrightarrow L^{2}\left(\mathbb{R}^{n}\right)$ is compact.

Weighted spaces are a customary tool when dealing with singular solutions. As in 2], we define the following weighted fractional Sobolev spaces. The weights we consider are powers of the distance to the boundary of $\Omega$. We introduce the notation

$$
\delta(x, y)=\min \{\operatorname{dist}(x, \partial \Omega), \operatorname{dist}(y, \partial \Omega)\}
$$


Let $s=m+\sigma$, with $m \in \mathbb{N}$ and $\sigma \in(0,1)$, then

$$
H_{\alpha}^{s}(\Omega)=\left\{v \in H^{m}(\Omega):\left|D^{\beta} v\right|_{H_{\alpha}^{\sigma}(\Omega)}<\infty \forall \beta \in \mathbb{N}^{n} \text { s.t. }|\beta|=m\right\},
$$

where

$$
|w|_{H_{\alpha}^{\sigma}(\Omega)}=\iint_{\Omega \times \Omega} \frac{|w(x)-w(y)|^{2}}{|x-y|^{n+2 \sigma}} \delta(x, y)^{2 \alpha} d x d y .
$$

We equip this space with the norm

$$
\|v\|_{H_{\alpha}^{s}(\Omega)}^{2}=\|v\|_{H^{m}(\Omega)}^{2}+\sum_{|\beta|=m}\left|D^{\beta} v\right|_{H_{\alpha}^{\sigma}(\Omega)} .
$$

We also need to define spaces over $\mathbb{R}^{n}$. The global weighted Sobolev space $H_{\alpha, \Omega}^{s}\left(\mathbb{R}^{n}\right)$ is

$$
H_{\alpha, \Omega}^{s}\left(\mathbb{R}^{n}\right)=\left\{v \in H^{m}\left(\mathbb{R}^{n}\right):\left|D^{\beta} v\right|_{H_{\alpha, \Omega}^{\sigma}\left(\mathbb{R}^{n}\right)}<\infty \forall \beta \in \mathbb{N}^{n} \text { s.t. }|\beta|=m\right\},
$$

where

$$
|w|_{H_{\alpha, \Omega}^{\sigma}\left(\mathbb{R}^{n}\right)}=\iint_{\mathbb{R}^{n} \times \mathbb{R}^{n}} \frac{|w(x)-w(y)|^{2}}{|x-y|^{n+2 \sigma}} \delta(x, y)^{2 \alpha} d x d y .
$$

The norm on this space is

$$
\|v\|_{H_{\alpha, \Omega}^{s}\left(\mathbb{R}^{n}\right)}^{2}=\|v\|_{H^{m}\left(\mathbb{R}^{n}\right)}^{2}+\sum_{|\beta|=m}\left|D^{\beta} v\right|_{H_{\alpha, \Omega}^{\sigma}\left(\mathbb{R}^{n}\right)} .
$$

Whenever the set $\Omega$ is clear from the context, we drop the reference to it in the global case and simply write $H_{\alpha}^{s}\left(\mathbb{R}^{n}\right)$.

Remark 2.3. Although we are interested in the case $\alpha \geq 0$, we recall that in the definition of weighted Sobolev spaces $H_{\alpha}^{m}(\Omega)$, with $m$ being a nonnegative integer, arbitrary powers of $\delta(x)$ can be considered 34, Theorem 3.6]. On the other hand, for general weights some restrictions must be taken into account in order to get an adequate definition of the spaces, namely, to ensure their completeness. A classical family of weights is that of the Muckenhoupt $A_{2}$ class. In the global version $H_{\alpha}^{s}\left(\mathbb{R}^{n}\right)$ we need to restrict the range of $\alpha$ to $|\alpha|<1 / 2$ in order to have $\delta^{2 \alpha} \in A_{2}$.

2.2. Eigenvalue problem. In the sequel, we work within the Hilbert space

$$
\left(\mathbb{V},\|\cdot\|_{\mathbb{V}}\right):=\left(\widetilde{H}^{s}(\Omega), \sqrt{C(n, s)}|\cdot|_{H^{s}\left(\mathbb{R}^{n}\right)}\right) .
$$

In [49, the authors prove that for any $k \in \mathbb{N}$ the eigenvalues of 1.2 can be characterized as follows:

$$
\lambda^{(k)}=\min \left\{\frac{\|u\|_{\mathbb{V}}^{2}}{\|u\|_{L^{2}(\Omega)}^{2}}: u \in \mathbb{V}^{(k)} \backslash\{0\}\right\},
$$

where $\mathbb{V}^{(1)}=\mathbb{V}$ and

$$
\mathbb{V}^{(k)}:=\left\{u \in \mathbb{V}:\left\langle u, u^{(j)}\right\rangle=0 \quad \forall j=1, \ldots, k-1\right\}
$$

for all $k \geq 2$. Therefore, by the min-max theorem,

$$
\lambda^{(k)}=\min _{E \in S^{(k)}} \max _{u \in E} \frac{\|u\|_{\mathbb{V}}^{2}}{\|u\|_{L^{2}(\Omega)}^{2}}
$$

where $S^{(k)}$ denotes the set of all $k$-dimensional subspaces of $\mathbb{V}$. 
The first eigenvalue $\lambda^{(1)}$ is simple (see, for example, [49]). We now state some regularity properties of the eigenfunctions.

2.3. Regularity results. Given a function $f \in H^{r}(\Omega)(r \geq-s)$, let us consider the homogeneous Dirichlet problem for the fractional Laplacian,

$$
\left\{\begin{aligned}
(-\Delta)^{s} u=f & \text { in } \Omega, \\
u=0 & \text { in } \Omega^{c} .
\end{aligned}\right.
$$

Existence and uniqueness of a weak solution $u \in \widetilde{H}^{s}(\Omega)$ of the above equation is an immediate consequence of the Lax-Milgram lemma. We are interested in regularity estimates for such solution in standard and graded Sobolev spaces. In [2], these are obtained in terms of the Hölder regularity of the data.

Proposition 2.4 (See [2]). Let $\Omega$ be a Lipschitz domain satisfying the exterior ball condition and consider $\beta=1 / 2-s$ if $s<1 / 2$ or $\beta>0$ if $s \geq 1 / 2$. Then, if $f \in C^{\beta}(\Omega)$ for every $\varepsilon>0$, the solution $u$ of 2.4 belongs to $\widetilde{H}^{s+\frac{1}{2}-\varepsilon}(\Omega)$, with

$$
\|u\|_{\widetilde{H}^{s+\frac{1}{2}-\varepsilon}(\Omega)} \leq \frac{C(\Omega, s, n)}{\varepsilon}\|f\|_{C^{\beta}(\Omega)} .
$$

Moreover, if $s>1 / 2$ and $f \in C^{1-s}(\Omega)$, then for every $\varepsilon>0$, it holds that $u \in H_{\frac{1}{2}-\varepsilon}^{s+1-2 \varepsilon}(\Omega)$, with

$$
\|u\|_{H_{1 / 2-\varepsilon}^{s+1-2 \varepsilon}(\Omega)} \leq \frac{C(\Omega, s, n)}{\varepsilon}\|f\|_{C^{1-s}(\Omega)} .
$$

On the other hand, smoothness of eigenfunctions is deduced from the regularity theory for the fractional Laplacian. See [14, 44, 48, 51, 54].

Proposition 2.5. If $\Omega$ is a Lipschitz domain satisfying the exterior ball condition then any solution of 1.1 is in $C^{\infty}(\Omega) \cap L^{\infty}(\Omega)$.

Sobolev regularity of eigenfunctions is a consequence of the two previous propositions.

Proof of Proposition 1.3. Since $u \in C^{\infty}(\Omega)$ (cf. Proposition 2.5), the claim follows easily applying Proposition 2.4 .

Following Grubb 30, it is also possible to obtain Sobolev regularity results for the solution to (2.4) in terms of Sobolev regularity of the right hand side. In that paper, the author deals with Hörmander $\mu$-spaces $H_{p}^{\mu(s)}$; see that work for a definition and further details. The following result is a particular case of Theorem 7.1 therein:

Theorem 2.6. Let $\Omega$ be a smooth domain, $\ell>s-1 / 2$ and assume $u \in \tilde{H}^{\sigma}(\Omega)$ for some $\sigma>s-1 / 2$ and consider a right hand side function $f \in H^{\ell-2 s}(\Omega)$. Then, it holds that $u \in H^{s(\ell)}(\Omega)$.

In particular, considering $\ell=r+2 s$ in the previous theorem and taking into account that

$$
H^{s(r+2 s)}(\bar{\Omega}) \begin{cases}=\widetilde{H}^{2 s+r}(\Omega) & \text { if } 0<s+r<1 / 2, \\ \subset \widetilde{H}^{s+1 / 2-\varepsilon}(\Omega) \forall \varepsilon>0, & \text { if } 1 / 2 \leq s+r<1,\end{cases}
$$

(see [30, Theorem 5.4]), we obtain: 
Proposition 2.7. Let $\Omega$ be a smooth domain, $f \in H^{r}(\Omega)$ for $r \geq-s$ and $u \in$ $\widetilde{H}^{s}(\Omega)$ be the solution of the Dirichlet problem 2.4. Then, the following regularity estimate holds

$$
|u|_{H^{s+\alpha}\left(\mathbb{R}^{n}\right)} \leq C(n, \alpha)\|f\|_{H^{r}(\Omega)} .
$$

Here, $\alpha=s+r$ if $s+r<1 / 2$ and $\alpha=1 / 2-\varepsilon$ if $s+r \geq 1 / 2$, with $\varepsilon>0$ arbitrarily small.

Remark 2.8. Assuming further Sobolev regularity in the right hand side function does not imply that the solution will be any smoother than what is given by the previous proposition. Indeed, if $f \in H^{r}(\Omega)$, then Theorem 2.6 gives $u \in H^{s(r+2 s)}(\Omega)$, which can not be embedded in any space sharper than $H^{s+1 / 2-\varepsilon}(\Omega)$ if $r+s \geq 1 / 2$.

Moreover, other regularity estimates for eigenfunctions of the fractional Laplacian in smooth domains are derived in [31, 45]. These estimates are formulated in terms of Hölder norms. Letting $d$ be a smooth function that behaves like dist $(x, \partial \Omega)$ near the boundary of $\Omega$, it is shown that any eigenfunction $u$ of 1.1 lies in the space $d^{s} C^{2 s(-\varepsilon)}(\bar{\Omega})$, where the $\varepsilon$ is active only if $s=1 / 2$ and that $u / d^{s}$ does not vanish near $\partial \Omega$. This shows that no further regularity than $H^{s+1 / 2-\varepsilon}(\Omega)$ should be expected for eigenfunctions.

2.4. Finite element approximations. Let $\mathcal{T}_{h}$ be a family of shape-regular triangulations of $\Omega$ (see introduction). Observe that for all $h>0$ the discrete space $\mathbb{V}_{h}$ is a subset of the continuous space.

We have the analogue min-max characterization for eigenvalues of the discrete problem,

$$
\lambda_{h}^{(k)}=\min _{E \in S_{h}^{(k)}} \max _{u \in E} \frac{\|u\|_{\mathbb{V}}^{2}}{\|u\|_{L^{2}(\Omega)}^{2}},
$$

where $S_{h}^{(k)}$ denotes the set of all $k$ dimensional subspaces of $\mathbb{V}_{h}$.

Remark 2.9. It follows from $\mathbb{V}_{h} \subset \mathbb{V}$ that

$$
\lambda^{(k)} \leq \lambda_{h}^{(k)} .
$$

The second part of Proposition 1.3 is exploited by using finite element approximations on adequately graded meshes. This idea is standard, in problems with corner singularities or to cope with boundary layers arising in convection-dominated problems. The following construction of graded meshes is based on [29, Section 8.4]. We assume that in addition to being shape-regular, our sequence of meshes enjoys satisfies the following graded hypotheses. First, we pick an arbitrary mesh size parameter $h>0$ and define, for $\varepsilon$ small enough, a number $1 \leq \mu$. Then, we assume that for any $T \in \mathcal{T}_{h}$,

$$
\begin{array}{ll}
\text { if } T \cap \partial \Omega \neq \emptyset, & \text { then } h_{T} \leq C(\sigma) h^{\mu} ; \\
\text { otherwise, } & h_{T} \leq C(\sigma) h \operatorname{dist}(T, \partial \Omega)^{(\mu-1) / \mu} .
\end{array}
$$

Constructing graded meshes as above, finite element approximations to solutions of (2.4) were proved to deliver an enhanced order of convergence (see [2]).

Lastly, we want to mention that in the following sections we will consider a quasi-interpolation operator $I_{h}: H^{l}(\Omega) \rightarrow \mathbb{V}_{h}$ satisfying the following estimate: there exists $C>0$ such that for any $w \in H^{l}(\Omega)$,

$$
\left\|w-I_{h} w\right\|_{\mathbb{V}} \leq C h^{l-s}|w|_{H^{l}(\Omega)} .
$$


Quasi-interpolation operators were introduced in [17] (see also [4]), and an estimate like 2.5 is derived, for example, in [2].

\section{Convergence of eigenvalues And eigenfunctions in GaP Distance}

In this section we prove Theorem 1.2 , stating that the discrete eigenvalue problem (1.3) converges to the continuous 1.2 in the gap distance (recall the definition of convergence given in the introduction). We only assume that the domain $\Omega$ satisfies (1.4), so that the embedding $\mathbb{V} \hookrightarrow L^{2}\left(\mathbb{R}^{n}\right)$ is compact (cf. Proposition 2.2).

Let us start by defining the solution operators of the continuous and discrete problems, $T: L^{2}(\Omega) \rightarrow \mathbb{V}$ and $T_{h}: L^{2}(\Omega) \rightarrow \mathbb{V}_{h}$. Given $f \in L^{2}(\Omega)$, we define $T f \in \mathbb{V}$ as the unique solution of

$$
\langle T f, v\rangle=(f, v) \quad \forall v \in \mathbb{V},
$$

and $T_{h} f \in \mathbb{V}_{h}$ as the unique solution of

$$
\left\langle T_{h} f, v_{h}\right\rangle=\left(f, v_{h}\right) \quad \forall v_{h} \in \mathbb{V}_{h} .
$$

Observe that if $(u, \lambda)$ is an eigenpair, then $T(\lambda u)=u$ and $T_{h}(\lambda u)=\Pi_{h} u$.

To prove Theorem 1.2 , by [10, Proposition 7.4 and Remark 7.5], we only need to show that the operators $T$ and $T_{h}$ are compact and

$$
\left\|T-T_{h}\right\|_{\mathcal{L}\left(L^{2}(\Omega), \mathbb{V}\right)} \rightarrow 0 \text { as } h \rightarrow 0 .
$$

Lemma 3.1. The operators $T$ and $T_{h}$ are compact.

Proof. Let $\left\{f_{k}\right\}_{k \in \mathbb{N}}$ be a bounded sequence in $L^{2}(\Omega)$. Then, there exists a subsequence of $\left\{f_{k}\right\}_{k \in \mathbb{N}}$ (still denoted by $\left\{f_{k}\right\}$ ) and $f \in L^{2}(\Omega)$ such that $f_{k} \rightarrow f$ weakly in $L^{2}(\Omega)$. Taking $v=T f_{k}$ in (3.1), we get

$$
\left\|T f_{k}\right\|_{\mathbb{V}}^{2}=\left(f_{k}, T f_{k}\right) \leq C\left\|T f_{k}\right\|_{L^{2}(\Omega)} \quad \forall k \in \mathbb{N} .
$$

Therefore, by Poincaré inequality 2.1), we have that $\left\{T f_{k}\right\}_{k \in \mathbb{N}}$ is bounded in $\mathbb{V}$. Thus, there exists a subsequence of $\left\{f_{k}\right\}_{k \in \mathbb{N}}$ (still denoted by $\left\{f_{k}\right\}_{k \in \mathbb{N}}$ ) and $u \in \mathbb{V}$ such that $T f_{k} \rightarrow u$ weakly in $\mathbb{V}$. Hence

$$
\langle u, v\rangle=\lim _{k \rightarrow \infty}\left\langle T f_{k}, v\right\rangle=\lim _{k \rightarrow \infty}\left(f_{k}, v\right)=(f, v) \quad \forall v \in \mathbb{V}
$$

that is, $u=T f$.

On the other hand, since the inclusion $\mathbb{V} \hookrightarrow L^{2}\left(\mathbb{R}^{n}\right)$ is compact, passing if necessary to a subsequence we may assume

$$
\begin{aligned}
& T f_{k} \rightarrow T f \text { weakly in } \mathbb{V}, \\
& T f_{k} \rightarrow T f \text { strongly in } L^{2}\left(\mathbb{R}^{n}\right) .
\end{aligned}
$$

Then,

$$
\left\|T f_{k}\right\|_{\mathbb{V}}^{2}=\left(f_{k}, T f_{k}\right) \rightarrow(f, T f)=\|T f\|_{\mathbb{V}}^{2}
$$

as $n \rightarrow \infty$. Since the space $\mathbb{V}$ is uniformly convex, it follows that

$$
T f_{k} \rightarrow T f \text { strongly in } \mathbb{V} \text {. }
$$

For the operators $T_{h}$ the result follows since they are finite-rank operators. 
Lemma 3.2. The following norm convergence holds true:

$$
\left\|T-T_{h}\right\|_{\mathcal{L}\left(L^{2}(\Omega), \mathbb{V}\right)} \rightarrow 0 \text { as } h \rightarrow 0 .
$$

Proof. For each $h$, take $f_{h} \in L^{2}(\Omega)$ such that $\left\|f_{h}\right\|_{L^{2}(\Omega)}=1$ and

$$
\sup _{\|f\|_{L^{2}(\Omega)}=1}\left\|T f-T_{h} f\right\|_{\mathbb{V}}=\left\|T f_{h}-T_{h} f_{h}\right\|_{\mathbb{V}} .
$$

Then, to prove the result, it is enough to show that for any sequence $h_{k} \rightarrow 0$ there is a subsequence $\left\{h_{k_{j}}\right\}_{j \in \mathbb{N}}$ such that

$$
\left\|T f_{h_{k_{j}}}-T_{h_{k_{j}}} f_{h_{k_{j}}}\right\|_{\mathbb{V}} \rightarrow 0 \quad \text { as } j \rightarrow \infty \text {. }
$$

Let $\left\{h_{k}\right\}_{k \in \mathbb{N}}$ be a sequence sucht that $h_{k} \rightarrow 0$. It follows from $\left\|f_{h_{k}}\right\|_{L^{2}(\Omega)}=1$ for all $k \in \mathbb{N}$ that there exist a subsequence $\left\{f_{h_{k_{j}}}\right\}_{j \in \mathbb{N}}$ of $\left\{f_{h_{k}}\right\}_{k \in \mathbb{N}}$ and $f \in L^{2}(\Omega)$

such that $f_{h_{k_{j}}} \rightarrow f$ weakly in $L^{2}(\Omega)$. Proceeding as in the proof of Lemma 3.1 (and passing if necessary to a subsequence), we may assume

$$
\begin{aligned}
& T_{h_{k_{j}}} f_{h_{k_{j}}} \rightarrow v \text { weakly in } \mathbb{V}, \\
& T_{h_{k_{j}}} f_{h_{k_{j}}} \rightarrow v \text { strongly in } L^{2}\left(\mathbb{R}^{n}\right) .
\end{aligned}
$$

On the other hand, it follows from 2.5 that

$$
\begin{aligned}
& I_{h} \varphi \rightarrow \varphi \text { strongly in } \mathbb{V}, \\
& I_{h} \varphi \rightarrow \varphi \text { strongly in } L^{2}\left(\mathbb{R}^{n}\right),
\end{aligned}
$$

for any $\varphi \in C_{0}^{\infty}(\Omega)$. Therefore,

$$
\langle v, \varphi\rangle=\lim _{j \rightarrow \infty}\left\langle T_{h_{k_{j}}} f_{h_{k_{j}}}, I_{h_{k_{j}}} \varphi\right\rangle=\lim _{j \rightarrow \infty}\left(f_{h_{k_{j}}}, I_{h_{k_{j}}} \varphi\right)=(f, \varphi) \quad \forall \varphi \in C_{0}^{\infty}(\Omega),
$$

which means that $v=T f$. Then,

$$
\left\|T f_{h_{k_{j}}}-T_{h_{k_{j}}} f_{h_{k_{j}}}\right\|_{\mathbb{V}}^{2}=\left(f_{h_{k_{j}}}, T f_{h_{k_{j}}}-T_{h_{k_{j}}} f_{h_{k_{j}}}\right) \rightarrow 0 .
$$

Now we conclude the convergence of the discrete eigenvalue problem to the continuous in the gap distance.

Proof of Theorem 1.2. The proof follows by Lemmas 3.1 and 3.2 and using Proposition 7.4 and Remark 7.5 of [10].

\section{ORDER OF CONVERGENCE}

Assuming certain regularity on the domain $\Omega$, we are able to deduce orders of convergence of the finite element approximations. This is attained as an application of the Babuška-Osborn theory [6]; an important tool in this regards is given by considering approximation properties of $\Pi_{h}: \mathbb{V} \rightarrow \mathbb{V}_{h}$, the projection with respect to the $\|\cdot\|_{\mathbb{V}}$ norm. To be specific, given $u \in \mathbb{V}$, this is the only function in $\mathbb{V}_{h}$ such that the Galerkin orthogonality

$$
\left\langle u-\Pi_{h} u, v_{h}\right\rangle=0 \quad \forall v_{h} \in \mathbb{V}_{h}
$$

holds, or equivalently,

$$
\left\|u-\Pi_{h} u\right\|_{\mathbb{V}}=\inf _{v_{h} \in \mathbb{V}_{h}}\left\|u-v_{h}\right\|_{\mathbb{V}} .
$$


Observe that if $u$ is the solution of 2.4 , then $\Pi_{h} u$ corresponds to the solution of the corresponding discrete problem on $\mathbb{V}_{h}$.

Proposition 4.1. Let $\Omega$ be a Lipschitz domain satisfying the exterior ball condition and $u$ be an eigenfunction of $(1.2$. Then, for any $\varepsilon>0$ there exists a positive constant $C$ independent of $h$ such that

$$
\left\|u-\Pi_{h} u\right\|_{\mathbb{V}} \leq C h^{1 / 2-\varepsilon} .
$$

Also, if $s>1 / 2$, constructing meshes according to grading hypothesis ( $\mathrm{H}$ ) (setting the parameter $\mu$ equal to 2 ), it holds that

$$
\left\|u-\Pi_{h} u\right\|_{\mathbb{V}} \leq C h \sqrt{|\ln h|} .
$$

Proof. Upon considering estimate 4.1 and Proposition 1.3 , the proof follows as in [2, Theorem 4.7].

Remark 4.2. In view of the previous proposition, and applying the abstract theory from [6] Theorem 1.4 follows.

In the remainder of this section, we study convergence of discrete eigenfunctions in the $L^{2}$ norm. We first prove the $L^{2}$ convergence of the energy projection over the discrete space. Notice that smoothness of the domain is required in order to apply Proposition 2.7.

Proposition 4.3. Let $\Omega$ be a smooth domain and $u$ be an eigenfunction of 1.2 . Then, for any $\varepsilon>0$ there is a positive constant $C$ independent of $h$ such that

$$
\left\|u-\Pi_{h} u\right\|_{L^{2}(\Omega)} \leq C h^{1 / 2+\alpha-\varepsilon} .
$$

Here, $\alpha=s$ if $s<1 / 2$ and $\alpha=1 / 2-\varepsilon$ if $s \geq 1 / 2$.

Proof. We apply an Aubin-Nitsche duality argument. Let $w \in \mathbb{V}$ be the weak solution of the boundary value problem

$$
\left\{\begin{aligned}
(-\Delta)^{s} w & =u-\Pi_{h} u & & \text { in } \Omega \\
w & =0 & & \text { in } \Omega^{c} .
\end{aligned}\right.
$$

Then, resorting to Galerkin orthogonality again we obtain

$$
\left\|u-\Pi_{h} u\right\|_{L^{2}(\Omega)}^{2}=\left\langle w, u-\Pi_{h} u\right\rangle \leq\left\|w-I_{h} w\right\|_{\mathbb{V}}\left\|u-\Pi_{h} u\right\|_{\mathbb{V}}
$$

where $I_{h} w \in \mathbb{V}_{h}$ is the interpolator of $w$.

Taking into account the regularity given by Proposition 2.7 with $r=0$, interpolation estimate 2.5 gives

$$
\left\|w-I_{h} w\right\|_{\mathbb{V}} \leq C h^{\alpha}|w|_{H^{s+\alpha}(\Omega)} \leq C h^{\alpha}\left\|u-\Pi_{h} u\right\|_{L^{2}(\Omega)} .
$$

Finally, using the error estimate 4.2 we obtain

$$
\left\|u-\Pi_{h} u\right\|_{L^{2}(\Omega)}^{2} \leq C h^{1 / 2+\alpha-\varepsilon}|u|_{H^{s+1 / 2-\varepsilon}(\Omega)}\left\|u-\Pi_{h} u\right\|_{L^{2}(\Omega)},
$$

and then estimate 4.4 follows.

The proof of Theorem 1.5 follows as in [43, Lemma 6.4-3], using Proposition 4.3. We include a proof here for completeness. 
Proof of Theorem 1.5. In first place, we assume that $m=1$ since the case $m>1$ is similar (see [10, 43]). We define

$$
\omega_{h}^{(k)}:=\left(\Pi_{h} u^{(k)}, u_{h}^{(k)}\right) u_{h}^{(k)}
$$

and the quantity

$$
\rho_{h}^{(k)}:=\max _{i \neq k} \frac{\lambda^{(k)}}{\left|\lambda^{(k)}-\lambda_{h}^{(i)}\right|}
$$

Then

$$
\begin{aligned}
\left\|u^{(k)}-u_{h}^{(k)}\right\|_{L^{2}(\Omega)} \leq & \left\|u^{(k)}-\Pi_{h} u^{(k)}\right\|_{L^{2}(\Omega)} \\
& +\left\|\Pi_{h} u^{(k)}-\omega_{h}^{(k)}\right\|_{L^{2}(\Omega)}+\left\|\omega_{h}^{(k)}-u_{h}^{(k)}\right\|_{L^{2}(\Omega)} .
\end{aligned}
$$

We are going to estimate the terms in the right hand side separately.

Given $\varepsilon>0$, it follows from our regularity estimate (4.4) that there exists $C>0$ independent of $h$ such that

$$
\left\|u^{(k)}-\Pi_{h} u^{(k)}\right\|_{L^{2}(\Omega)} \leq C h^{\alpha+1 / 2-\varepsilon} .
$$

Moreover, since

$$
\left(\Pi_{h} u^{(k)}, u_{h}^{(i)}\right)=\frac{1}{\lambda_{h}^{(i)}}\left\langle\Pi_{h} u^{(k)}, u_{h}^{(i)}\right\rangle=\frac{1}{\lambda_{h}^{(i)}}\left\langle u^{(k)}, u_{h}^{(i)}\right\rangle=\frac{\lambda^{(k)}}{\lambda_{h}^{(i)}}\left(u^{(k)}, u_{h}^{(i)}\right),
$$

we have

$$
\left|\left(\Pi_{h} u^{(k)}, u_{h}^{(i)}\right)\right| \leq \rho_{h}^{(k)}\left|\left(u^{(k)}-\Pi_{h} u^{(k)}, u_{h}^{(i)}\right)\right| .
$$

So,

$$
\begin{aligned}
\left\|\Pi_{h} u^{(k)}-\omega_{h}^{(k)}\right\|_{L^{2}(\Omega)}^{2} & =\sum_{i \neq k}\left(\Pi_{h} u^{(k)}, u_{h}^{(i)}\right)^{2} \\
& \leq\left[\rho_{h}^{(k)}\right]^{2} \sum_{i \neq k}\left(u^{(k)}-\Pi_{h} u^{(k)}, u_{h}^{(i)}\right)^{2} \\
& \leq\left[\rho_{h}^{(k)}\right]^{2}\left\|u^{(k)}-\Pi_{h} u^{(k)}\right\|_{L^{2}(\Omega)}^{2} \leq C h^{\alpha+1 / 2-\varepsilon} .
\end{aligned}
$$

Finally, let us show that

$$
\left\|\omega_{h}^{(k)}-u_{h}^{(k)}\right\|_{L^{2}(\Omega)} \leq\left\|\omega_{h}^{(k)}-u^{(k)}\right\|_{L^{2}(\Omega)},
$$

so that

$$
\left\|\omega_{h}^{(k)}-u_{h}^{(k)}\right\|_{L^{2}(\Omega)} \leq\left\|u^{(k)}-\Pi_{h} u^{(k)}\right\|_{L^{2}(\Omega)}+\left\|\Pi_{h} u^{(k)}-\omega_{h}^{(k)}\right\|_{L^{2}(\Omega)} .
$$

Indeed, on one hand we have

$$
u_{h}^{(k)}-\omega_{h}^{(k)}=\left[1-\left(\Pi_{h} u^{(k)}, u_{h}^{(k)}\right)\right] u_{h}^{(k)} .
$$

On the other hand, due to the normalizations $\left\|u^{(k)}\right\|_{L^{2}(\Omega)}=\left\|u_{h}^{(k)}\right\|_{L^{2}(\Omega)}=1$, we have

$$
\left|1-\left\|\omega_{h}^{(k)}\right\|_{L^{2}(\Omega)}\right| \leq\left\|u^{(k)}-\omega_{h}^{(k)}\right\|_{L^{2}(\Omega)}
$$

and

$$
\left\|\omega_{h}^{(k)}\right\|_{L^{2}(\Omega)}=\left|\left(\Pi_{h} u^{(k)}, u_{h}^{(k)}\right)\right| .
$$


Therefore, if we choose the sign of $u_{h}^{(k)}$ in such a way that $\left(\Pi_{h} u^{(k)}, u_{h}^{(k)}\right) \geq 0$, we deduce

$$
\begin{aligned}
\left\|u_{h}^{(k)}-\omega_{h}^{(k)}\right\|_{L^{2}(\Omega)} & =\left|1-\left(\Pi_{h} u^{(k)}, u_{h}^{(k)}\right)\right| \\
& =|1-|\left(\Pi_{h} u^{(k)}, u_{h}^{(k)}\right)|| \\
& \leq\left\|u^{(k)}-\omega_{h}^{(k)}\right\|_{L^{2}(\Omega)},
\end{aligned}
$$

as stated in 4.9.

Thus, estimate 1.5 is obtained by combining $(4.6),(4.7),(4.8)$ and $(4.9)$.

\section{NumERICAL RESUlts}

This section exhibits the outcome of a variety of experiments carried out by the authors in one- and two-dimensional domains. Since in general no closed formula for the eigenvalues of the fractional Laplacian is available, we have estimated the order of convergence by means of a least-squares fitting of the model

$$
\lambda_{h}^{(k)}=\lambda^{(k)}+C h^{\alpha} .
$$

This allows to extrapolate approximations of the eigenvalues as well (in the tables we denote this extrapolated value of $\lambda^{(k)}$ as $\left.\lambda_{\text {ext }}^{(k)}\right)$.

Throughout this section, the results are compared with those available in the literature. In first place we consider one-dimensional problems, which have been widely studied both theoretically and from the numerical point of view. Next, we show some examples in two-dimensional domains: the unit ball, a square and an $L$-shaped domain. As for the ball, the deep results of 24] allow to obtain sharp estimates on the eigenvalues, and thus provide a point of comparison for the validity of the FE implementation. Regarding the square, some estimates for the eigenvalues are found in [36]. The main interest of the $L$-shaped domain is that, although it does not satisfy the "standard" requirements to regularity of eigenfunctions to hold, the numerical order of convergence is the same as in problems posed on smooth, convex domains. Finally, Subsection 5.3 is concerned with the computation of higher-order eigenspaces.

General estimates for eigenvalues, valid for a class of domains, are obtained by Chen and Song [16]. In that paper, the authors state upper and lower bounds for eigenvalues of the fractional Laplacian on domains satisfying the exterior cone condition. Calling $\mu^{(k)}$ the $k$-th eigenvalue of the Laplacian with Dirichlet boundary conditions on the domain $\Omega$, they prove that there exists a constant $C=C(\Omega)$ such that

$$
C\left(\mu^{(k)}\right)^{s} \leq \lambda^{(k)} \leq\left(\mu^{(k)}\right)^{s}
$$

If $\Omega$ is a bounded convex domain, then $C$ can be taken as $1 / 2$. It is noteworthy that, due to the scaling property of the fractional Laplacian, eigenvalues for the dilations of a domain $\Omega$ are obtained by means of $\lambda^{(k)}(\gamma \Omega)=\gamma^{-2 s} \lambda^{(k)}(\Omega)$.

Since we are working with conforming methods, as well as providing approximations, the discrete eigenvalues yield upper bounds for the corresponding continuous eigenvalues. This is of special interest in those cases in which theoretical estimates 
are not sharp, or the non-symmetry of the domain precludes the possibility of developing arguments such as the ones in 24.

5.1. One-dimensional intervals. Eigenvalues for the fractional Laplacian in intervals have been studied by other authors previously. In [57, a discretized model of the fractional Laplacian is developed, and a numerical study of eigenfunctions and eigenvalues is implemented for different boundary conditions. In [35, the authors deal with one dimensional problems for $s=1 / 2$, and provide asymptotic expansion for eigenvalues. Later, Kwaśnicki [36] extended this work to the whole range $s \in(0,1)$. Namely, he showed the following identity for the $k$-th eigenvalue in the interval $(-1,1)$

$$
\lambda^{(k)}=\left(\frac{k \pi}{2}-\frac{(1-s) \pi}{4}\right)^{2 s}+\frac{1-s}{\sqrt{s}} O\left(k^{-1}\right) .
$$

Moreover, in that work a method to obtain lower bounds in arbitrary bounded domains is developed, and it is proved that, on one spacial dimension, eigenvalues are simple if $s \geq 1 / 2$. As eigenvalues are simple and we are working in one dimension, it is not difficult to numerically estimate the order of convergence of eigenfunctions in the $L^{2}$-norm. Indeed, normalizing the discrete eigenfunctions so that $\left\|u_{h}^{(k)}\right\|_{L^{2}(-1,1)}=1$ and choosing their sign adequately, these are then compared with a solution on a very fine grid.

On the other hand, in 23 it is performed a numerical study of the fractional Schrödinger equation in an infinite potential well in one spacial dimension. The authors find numerically the ground and first excited states and their corresponding eigenvalues for the stationary linear problem, which corresponds to the first two eigenpairs of our equation (1.1).

In Table 5.1, our results for the first 2 eigenpairs are displayed, computed over a sequence of uniform meshes with $800,1600,3200$ and 6400 elements. The extrapolated numerical values are compared with the estimates from [23, 36]; the orders of convergence are in good agreement with those predicted correspondingly by Theorems 1.4 and 1.5 . Moreover, we illustrate the sharpness of Proposition 1.3 by displaying in Figure 5.1 the first two $L^{2}$-normalized eigenfunctions for $s=0.1$ and $s=0.9$. As predicted by Remark 2.8, these functions are smooth within the interval, but behave as $d(x, \partial \Omega)^{s}$ near the boundary of the domain.

5.2. Two-dimensional experiments. The theoretical order of convergence for eigenvalues is also attained in the following examples in $\mathbb{R}^{2}$. The implementation for these experiments is based on the code from [1.

Unit ball. Let us consider the fractional eigenvalue problem on the two-dimensional unit ball. In [25, the weighted operator $u \mapsto(-\Delta)^{s}\left(\omega^{s} u\right)$ is studied, where $\omega^{s}(x)=\left(1-|x|^{2}\right)_{+}^{s}$. In particular, explicit formulas for eigenvalues and eigenfunctions of this operator are established. Furthermore, in [24] the same authors exploit these expressions to obtain two-sided bounds for the eigenvalues of the fractional Laplacian in the unit ball in any dimension. This method provides sharp estimates; however, it depends on the decomposition of the fractional Laplacian as a weighted operator, and the weight $\omega^{s}$ is only explicitly known for the unit ball. 

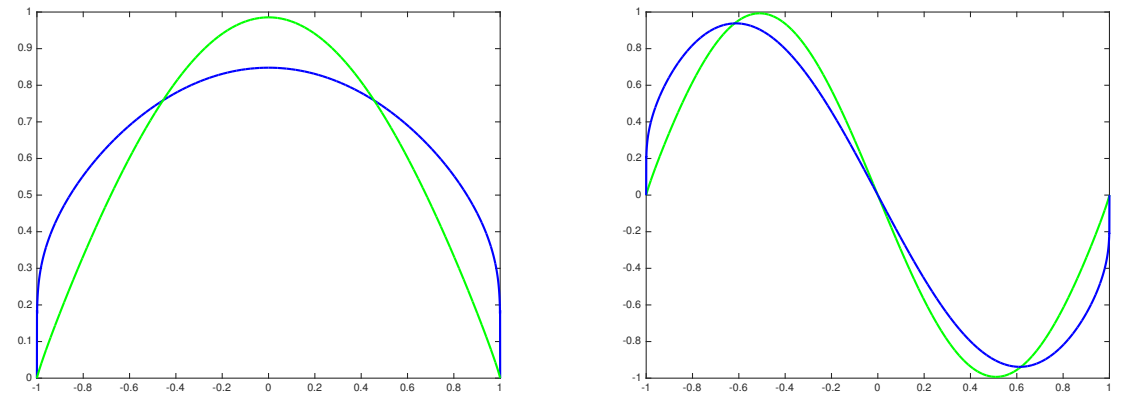

Figure 5.1. First (left panel) and second (right panel) $L^{2}$ normalized discrete eigenfunctions in the interval $(-1,1)$. Color blue corresponds to $s=0.1$, while green corresponds to $s=0.9$.

TABle 5.1. First 2 eigenpairs in the interval $(-1,1)$. On the left, the extrapolated numerical values are compared with the results from [23] and with approximation (5.2), obtained in [36]. On the right, orders of convergence for eigenvalues and eigenfunctions in the $L^{2}$-norm (obtained by a least-square fitting) are displayed.

\begin{tabular}{ccccccccccc}
\hline \multicolumn{1}{c}{ Numerical values } & \multicolumn{4}{c}{ Orders } \\
\hline$s$ & $\lambda_{\text {ext }}^{(1)}$ & $\lambda^{(1)}[23]$ & $\lambda^{(1)}[36]$ & $\lambda_{\text {ext }}^{(2)}$ & $\lambda^{(2)}[23]$ & $\lambda^{(2)}[36]$ & $\lambda^{(1)}$ & $\lambda^{(2)}$ & $u^{(1)}$ & $u^{(2)}$ \\
\hline 0.05 & 0.9726 & 0.9726 & 0.9809 & 1.0922 & 1.0922 & 1.0913 & 1.108 & 1.149 & 0.551 & 0.568 \\
0.1 & 0.9575 & 0.9575 & 0.9712 & 1.1965 & 1.1966 & 1.1948 & 1.071 & 1.102 & 0.612 & 0.625 \\
0.25 & 0.9702 & 0.9702 & 0.9908 & 1.6015 & 1.6016 & 1.5977 & 1.021 & 1.038 & 0.762 & 0.782 \\
0.5 & 1.1577 & 1.1578 & 1.1781 & 2.7548 & 2.7549 & 2.7488 & 1.001 & 0.979 & 0.961 & 0.969 \\
0.75 & 1.5975 & 1.5976 & 1.6114 & 5.0598 & 5.0600 & 5.0545 & 0.998 & 0.999 & 0.998 & 0.998 \\
0.9 & 2.0487 & - & 2.0555 & 7.5031 & - & 7.5003 & 1.004 & 1.021 & 0.999 & 0.999 \\
0.95 & 2.2481 & 2.2441 & 2.2477 & 8.5958 & 8.5959 & 8.5942 & 1.035 & 1.142 & 0.999 & 0.999 \\
\hline
\end{tabular}

In Table 5.2, our results for the first eigenvalue are compared with those of 24] for different values of $s$, computed over a family of uniform meshes with mesh sizes $h \in\{1 / 30,1 / 35,1 / 40,1 / 45,1 / 50\}$. This comparison serves as a test for the validity of the code we are employing. As well as the extrapolated value of $\lambda^{(1)}$ and the numerical order of convergence, for every $s$ considered we exhibit an upper bound for the first eigenvalue. These outcomes are consistent with those from [24] and the theoretical order of convergence given by Theorem 1.4 .

Computations with graded meshes were carried out for this domain as well. The grading parameter $\mu$ in $(\mathrm{H})$ was set to be equal to 2 , and meshes were taken with about the same total of degrees of freedom as in the experiments with uniform meshes. For a description of how to build these graded meshes, we refer the reader to Section 5.2 in [2]. In Table 5.3 we summarize our findings. For $s \geq 1 / 2$, we estimated the order of convergence towards the first eigenvalue both with uniform and graded meshes, and also compared the extrapolated value of this eigenvalue. An increase in the convergence rate, in agreement with Theorem 1.4 , is observed. 
TABLe 5.2. First eigenvalue in the unit ball in $\mathbb{R}^{2}$. Estimate from 24]; extrapolated value of $\lambda^{(1)}$; upper bound obtained by the finite element method with a meshsize $h \sim 0.02$; numerical order of convergence.

\begin{tabular}{ccccc}
\hline$s$ & $\lambda^{(1)}$ & $\lambda_{\text {ext }}^{(1)}$ & $\lambda_{h}^{(1)}(\mathrm{UB})$ & Order \\
\hline 0.005 & 1.00475 & 1.00475 & 1.00480 & 0.9462 \\
0.05 & 1.05095 & 1.05094 & 1.05145 & 0.9455 \\
0.25 & 1.34373 & 1.34367 & 1.34626 & 0.9497 \\
0.5 & 2.00612 & 2.00607 & 2.01060 & 0.9686 \\
0.75 & 3.27594 & 3.27632 & 3.28043 & 1.0092 \\
\hline
\end{tabular}

TABle 5.3. Computational results in the unit ball in $\mathbb{R}^{2}$, for uniform and graded meshes. Orders of convergence are stated in terms of the mesh parameter $h$; this behaves like $N^{-1 / 2}, N$ being the number of nodes.

\begin{tabular}{ccccc}
\hline$s$ & Order (unif.) & Order (graded) & $\lambda_{\text {ext }}^{(1)}$ (unif.) & $\lambda_{\text {ext }}^{(1)}$ (graded) \\
\hline 0.5 & 0.9686 & 2.1528 & 2.0061 & 2.0061 \\
0.6 & 0.9808 & 2.1720 & 2.4165 & 2.4165 \\
0.7 & 0.9969 & 2.1066 & 2.9506 & 2.9507 \\
0.8 & 1.0348 & 2.0497 & 3.6494 & 3.6498 \\
0.9 & 1.1654 & 2.0943 & 4.5691 & 4.5695 \\
\hline
\end{tabular}

The grading parameter $\mu=2$ is optimal for every $s$. Indeed, this parameter is in correspondence with the weight in the regularity estimate from Proposition 1.3 . In this sense, the greater $\mu$ is, the greater the weight can be taken, and thus, the greater the differentiability order of solutions is. Therefore, increasing $\mu$ leads to an increment on the order of convergence with respect to the mesh size parameter. However, if $\mu>2$ this effect is compensated by the growth in the number of degrees of freedom. We refer the reader to $[12$ for details.

Square. Eigenvalue estimates for the case in which the domain $\Omega$ is a square in $\mathbb{R}^{2}$ were also addressed in [36. However, in order to obtain upper bounds, the method proposed in that work depends on having pointwise bounds of the Green function for the fractional Laplacian on $\Omega$. The estimates from [16, 36] are compared with our results in Table 5.4, where numerical orders of convergence are also displayed. The computations were carried over a sequence of unstructured uniform meshes with sizes $h \sim\{0.1,0.08,0.06,0.04\}$. The upper bound displayed in Table 5.4 corresponds to the computed result over the finest mesh in this sequence.

L-shaped domain. To the authors knowledge, there is no efficient method to estimate eigenvalues of the fractional Laplacian if the domain $\Omega$ lacks symmetry. The bound (5.1) remains valid as long as $\Omega$ satisfies the assumptions required, but the range that estimate provides is quite wide. 
TABle 5.4. First eigenvalue in the square $[-1,1]^{2}$. Best lower (LB) and upper (UB) bounds known before; upper bound obtained by the finite element method with a meshsize $h \sim 0.04$; extrapolated value of $\lambda^{(1)}$; numerical order of convergence.

\begin{tabular}{cccccc}
\hline$s$ & $\lambda^{(1)}(\mathrm{LB})$ & $\lambda^{(1)}(\mathrm{UB})$ & $\lambda_{h}^{(1)}(\mathrm{UB})$ & $\lambda_{\text {ext }}^{(1)}$ & Order \\
\hline 0.05 & $1.0308^{b}$ & $1.0831^{a}$ & 1.0412 & 1.0405 & 0.9229 \\
0.1 & $1.0506^{b}$ & $1.1731^{a}$ & 1.0895 & 1.0882 & 0.9230 \\
0.25 & $1.1587^{b}$ & $1.4905^{a}$ & 1.2844 & 1.2813 & 0.9283 \\
0.5 & $1.3844^{b}$ & $2.2214^{a}$ & 1.8395 & 1.8344 & 0.9622 \\
0.75 & $1.6555^{a}$ & $3.3109^{a}$ & 2.8921 & 2.8872 & 0.9940 \\
0.9 & $2.1034^{a}$ & $4.2067^{a}$ & 3.9492 & 3.9467 & 1.0654 \\
0.95 & $2.2781^{a}$ & $4.5562^{a}$ & 4.4083 & 4.4062 & 1.1496 \\
\hline \multicolumn{2}{c}{ See [16. ${ }^{b}$ See [36. } & & & &
\end{tabular}

The main advantage of employing the finite element method is that it is flexible enough to cope with a variety of domains. Moreover, as we are working with conforming approximations, sharp upper bounds for the eigenvalues may be obtained by considering discrete solutions on refined meshes.

In Proposition 1.3 which states that eigenfunctions belong to $\widetilde{H}^{s+1 / 2-\varepsilon}(\Omega)$, it was assumed that the domain $\Omega$ satisifies the exterior ball condition. For the Laplacian, in order to prove regularity of solutions, it is customary to assume that $\Omega$ is either smooth or at least convex. In those cases, it is well known that if $f \in H^{r}(\Omega)$ for some $r$, then the solutions of the Dirichlet problem with right hand side $f$ belong to $H^{r+2}(\Omega)$. However, if the domain has a re-entrant corner, solutions are less regular. This also applies to eigenfunctions: in the $L$-shaped domain $\Omega=[-1,1]^{2} \backslash[0,1]^{2}$, the first eigenvalue of the Laplacian is known not to belong to $H^{3 / 2}(\Omega)$.

Surprisingly, numerical evidence indicates that eigenvalues of the fractional Laplacian on this $L$-shaped domain converge with the same order as in the previous examples. This motivates us to conjecture that eigenfunctions and solutions to the Dirichlet equation (2.4) have the same Sobolev regularity than in smooth domains.

Our findings for the first eigenvalue, computed over unstructured, uniform meshes with sizes $h \sim\{0.1,0.08,0.06,0.04\}$, are summarized in Table 5.5

5.3. Approximation of high-order eigenspaces. In Theorems 1.4 and 1.5 we showed convergence rates of the discrete eigenpairs with respect to the meshsize. The constants involved in the convergence estimates depend on the domain, on $s$, the mesh regularity parameter $\sigma$ (cf. (Regularity)), and importantly, on the eigenvalue number $k$.

We refer to eigenvalues $\lambda_{k}$ corresponding to a 'large' $k$ as high-order eigenvalues. We point out that high-order eigenvalues need not to be large in magnitude; it follows from (5.1) that, for every $k, \lim _{s \rightarrow 0} \lambda_{s}^{(k)}=1$, where $\lambda_{s}^{(k)}$ denotes the $k$-th eigenvalue of the fractional Laplacian of order $s$. Nevertheless, for a fixed discretization, the quality of the approximation of the $k$-th eigenspace deteriorates as $k$ grows; the discrete system cannot approximate more eigenvalues than the number of degrees of freedom, and the finer-scale oscillations corresponding to high-order 
TABLE 5.5. First eigenvalues in the $L$-shaped domain $[-1,1]^{2} \backslash$ $[0,1]^{2}$. Upper bound obtained by the finite element method with a meshsize $h \sim 0.04$; extrapolated value of $\lambda^{(1)}$; numerical order of convergence.

\begin{tabular}{cccc}
\hline$s$ & $\lambda_{h}^{(1)}(\mathrm{UB})$ & $\lambda_{\text {ext }}^{(1)}$ & Order $\lambda^{(1)}$ \\
\hline 0.1 & 1.1434 & 1.1413 & 0.9085 \\
0.2 & 1.3386 & 1.3342 & 0.9103 \\
0.3 & 1.6025 & 1.5956 & 0.9160 \\
0.4 & 1.9593 & 1.9499 & 0.9267 \\
0.5 & 2.4440 & 2.4322 & 0.9459 \\
0.6 & 3.1072 & 3.0936 & 0.9812 \\
0.7 & 4.0228 & 4.0069 & 0.9822 \\
0.8 & 5.2994 & 5.2831 & 1.0609 \\
0.9 & 7.0975 & 7.0790 & 1.1891 \\
\hline
\end{tabular}

TABLE 5.6. One-hundredth eigenvalue in the interval $(-1,1)$, computed over a uniform partition of the interval with 8000 nodes. We compare our approximation with the asymptotic estimate $[5.2]$.

\begin{tabular}{cccc}
\hline$s$ & $\lambda_{h}^{(100)}$ & $\lambda^{(100)}[36]$ & Relative difference \\
\hline 0.05 & 1.65735 & 1.65732 & $2.3054 \times 10^{-5}$ \\
0.1 & 2.74690 & 2.74683 & $2.6087 \times 10^{-5}$ \\
0.25 & 12.5100 & 12.5096 & $3.2075 \times 10^{-5}$ \\
0.5 & 156.681 & 156.687 & $3.9854 \times 10^{-5}$ \\
0.75 & 1965.06 & 1965.01 & $2.3987 \times 10^{-5}$ \\
0.9 & 8966.95 & 8966.54 & $4.6387 \times 10^{-5}$ \\
0.95 & 14874.9 & 14873.8 & $7.4828 \times 10^{-5}$ \\
\hline
\end{tabular}

eigenvalues cannot be well captured by a coarse mesh. A relevant question that arises is, for a fixed $k$, how many degrees of freedom are needed to provide an approximation of the $k$-th eigenspace within a given tolerance.

The examples considered in subsections 5.1 and 5.2 illustrate the order of convergence obtained in theory by examining the first eigenvalues. Here, we provide numerical examples of the computation of higher-order eigenvalues. In Table 5.6, we compute the difference between the finite element approximation of $\lambda_{h}^{(100)}$ and the asymptotic estimate $(5.2)$ in the interval $(-1,1)$. We observe that, independently of the value of $s$, the finite element solutions with 8000 degrees of freedom offer approximations within a relative difference of about $5 \times 10^{-5}$ with respect to the asymptotic estimate. Since the relative error of the approximation 5.2 is of the order of $k^{-(1+2 s)}$, we deduce that, roughly, the relative error of the finite element approximations of $\lambda^{(100)}$ have a relative error of the order of $10^{-(2+4 s)}$ if $s \leq 3 / 4$ and of the order of $10^{-5}$ if $s>3 / 4$.

Computation of high-order eigenvalues is also feasible in more complex geometries. In the experiments carried out over the $L$-shaped domain $[-1,1]^{2} \backslash[0,1]^{2}$, 
it was observed that the eigenvalue $\lambda_{h}^{(91)}$ is simple, independently of $s$. Figure 5.2 (top) displays $L^{2}$-normalized eigenfunctions for $s=0.05$ and $s=0.95$ respectively, computed on a mesh with 11120 elements $(h \sim 0.04)$. Even though these functions seem to have a similar qualitative behavior, an important difference becomes apparent when inspecting cross sections of these plots (cf. Figure 5.2 (bottom)): the eigenfunction corresponding to $s=0.05$ exhibits steeper gradients near the boundary of the domain. The boundary behavior predicted by the regularity theory discussed in Subsection 2.3 extends robustly to high-order eigenvalues.
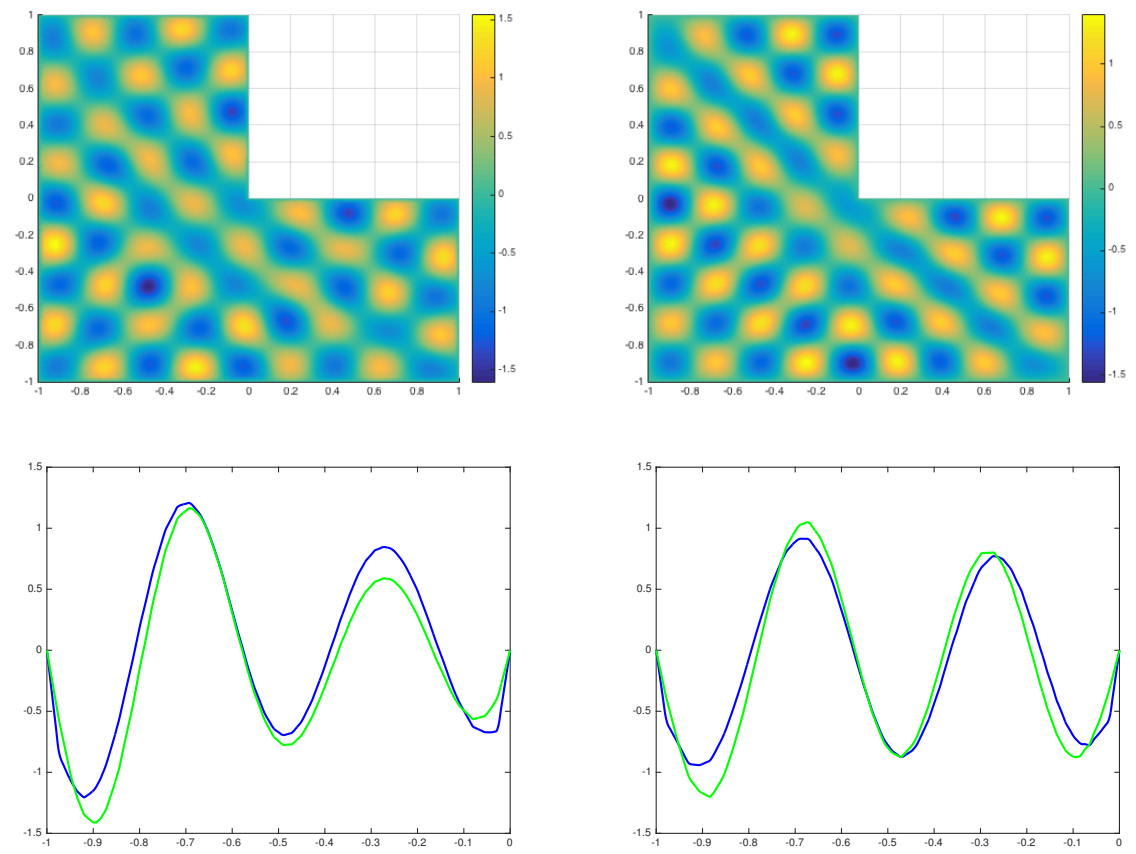

Figure 5.2. Finite element approximations of $u^{(91)}$ in the domain $[-1,1]^{2} \backslash[0,1]^{2}$. Top: plots of $u_{h}^{(91)}$ for $s=0.05$ (left) and $s=0.05$ (right), respectively. Bottom: cross sections at $y=0$ (left) and $y=0.4$; color blue corresponds to $s=0.05$ whereas color green corresponds to 0.95 .

Acknowledgments. The authors would like to thank G. Grubb and R. Rodríguez for the valuable help they provided through clarifying discussions on the topic of this paper, and to F. Bersetche for improving the efficiency of the Matlab code employed.

\section{REFERENCES}

[1] Gabriel Acosta, Francisco Bersetche, and Juan Pablo Borthagaray. A short FEM implementation for a $2 \mathrm{~d}$ homogeneous Dirichlet problem of a fractional Laplacian. Comput. Math. Appl., 74(4):784-816, 2017. 
[2] Gabriel Acosta and Juan Pablo Borthagaray. A fractional Laplace equation: Regularity of solutions and finite element approximations. SIAM Journal on Numerical Analysis, 55(2):472495, 2017.

[3] M. Ainsworth and C. Glusa. Towards an efficient finite element method for the integral fractional Laplacian on polygonal domains. arXiv:1708.01923v1, 2017.

[4] Paolo Amore, Francisco M Fernández, Christoph P Hofmann, and Ricardo A Sáenz. Collocation method for fractional quantum mechanics. Journal of Mathematical Physics, 51(12):122101, 2010.

[5] Xavier Antoine, Qinglin Tang, and Yong Zhang. On the ground states and dynamics of space fractional nonlinear Schrödinger/Gross-Pitaevskii equations with rotation term and nonlocal nonlinear interactions. Journal of Computational Physics, 325:74-97, 2016.

[6] I. Babuška and J. Osborn. Eigenvalue problems. In Handbook of numerical analysis, Vol. II, Handb. Numer. Anal., II, pages 641-787. North-Holland, Amsterdam, 1991.

[7] Weizhu Bao, Xinran Ruan, Jie Shen, and Changtao Sheng. Fundamental gaps of the fractional Schrödinger operator. arXiv preprint arXiv:1801.06517, 2018.

[8] David A. Benson, Stephen W. Wheatcraft, and Mark M. Meerschaert. Application of a fractional advection-dispersion equation. Water Resources Research, 36(6):1403-1412, 2000.

[9] Jean Bertoin. Lévy processes, volume 121 of Cambridge Tracts in Mathematics. Cambridge University Press, Cambridge, 1996.

[10] Daniele Boffi. Finite element approximation of eigenvalue problems. Acta Numer., 19:1-120, 2010.

[11] Daniele Boffi, Franco Brezzi, and Lucia Gastaldi. On the problem of spurious eigenvalues in the approximation of linear elliptic problems in mixed form. Math. Comp., 69(229):121-140, 2000.

[12] Andrea Bonito, Juan Pablo Borthagaray, Ricardo H. Nochetto, Enrique Otarola, and Abner J Salgado. Numerical methods for fractional diffusion. Computing and Visualization in Science. To appear., 2018.

[13] A. Buades, B. Coll, and J. M. Morel. Image denoising methods. A new nonlocal principle. SIAM Rev., 52(1):113-147, 2010. Reprint of "A review of image denoising algorithms, with a new one" [MR2162865].

[14] Luis Caffarelli and Luis Silvestre. Regularity theory for fully nonlinear integro-differential equations. Comm. Pure Appl. Math., 62(5):597-638, 2009.

[15] Peter Carr, Hélyette Geman, Dilip B. Madan, and Marc Yor. The fine structure of asset returns: An empirical investigation. The Journal of Business, 75(2):305-332, 2002.

[16] Zhen-Qing Chen and Renming Song. Two-sided eigenvalue estimates for subordinate processes in domains. J. Funct. Anal., 226(1):90-113, 2005.

[17] $\mathrm{Ph}$. Clément. Approximation by finite element functions using local regularization. Rev. Française Automat. Informat. Recherche Opérationnelle Sér. RAIRO Analyse Numérique, 9(R-2):77-84, 1975.

[18] Rama Cont and Peter Tankov. Financial modelling with jump processes. Chapman \& Hall/CRC Financial Mathematics Series. Chapman \& Hall/CRC, Boca Raton, FL, 2004.

[19] John H. Cushman and T.R. Ginn. Nonlocal dispersion in media with continuously evolving scales of heterogeneity. Transport in Porous Media, 13(1):123-138, 1993.

[20] Leandro M. Del Pezzo and Alexander Quaas. Global bifurcation for fractional $p$-Laplacian and an application. Z. Anal. Anwend., 35(4):411-447, 2016.

[21] Françoise Demengel and Gilbert Demengel. Functional spaces for the theory of elliptic partial differential equations. Universitext. Springer, London; EDP Sciences, Les Ulis, 2012. Translated from the 2007 French original by Reinie Erné.

[22] Eleonora Di Nezza, Giampiero Palatucci, and Enrico Valdinoci. Hitchhiker's guide to the fractional Sobolev spaces. Bull. Sci. Math., 136(5):521-573, 2012.

[23] Siwei Duo and Yanzhi Zhang. Computing the ground and first excited states of the fractional Schrödinger equation in an infinite potential well. Commun. Comput. Phys., 18(2):321-350, 2015.

[24] B. Dyda, A. Kuznetsov, and M. Kwaśnicki. Eigenvalues of the fractional Laplace operator in the unit ball. J. Lond. Math. Soc., 95(2):500-518, 2017.

[25] Bartłomiej Dyda, Alexey Kuznetsov, and Mateusz Kwaśnicki. Fractional Laplace operator and Meijer G-function. Constructive Approximation, 45(3):427-448, 2017. 
[26] P. Gatto and J.S. Hesthaven. Numerical approximation of the fractional Laplacian via hpfinite elements, with an application to image denoising. J. Sci. Comp., 65(1):249-270, 2015.

[27] Paolo Ghelardoni and Cecilia Magherini. A matrix method for fractional Sturm-Liouville problems on bounded domain. Advances in Computational Mathematics, 43(6):1377-1401, 2017.

[28] Guy Gilboa and Stanley Osher. Nonlocal operators with applications to image processing. Multiscale Model. Simul., 7(3):1005-1028, 2008.

[29] P. Grisvard. Elliptic problems in nonsmooth domains, volume 24 of Monographs and Studies in Mathematics. Pitman (Advanced Publishing Program), Boston, MA, 1985.

[30] Gerd Grubb. Fractional laplacians on domains, a development of Hörmander's theory of $\mu$-transmission pseudodifferential operators. Advances in Mathematics, 268:478 - 528, 2015.

[31] Gerd Grubb. Spectral results for mixed problems and fractional elliptic operators. J. Math. Anal. Appl., 421(2):1616-1634, 2015.

[32] Tosio Kato. Perturbation theory for linear operators. Classics in Mathematics. SpringerVerlag, Berlin, 1995. Reprint of the 1980 edition.

[33] Joseph Klafter and Igor M. Sokolov. Anomalous diffusion spreads its wings. Physics world, 18(8):29, 2005.

[34] Alois Kufner. Weighted Sobolev spaces. A Wiley-Interscience Publication. John Wiley \& Sons, Inc., New York, 1985. Translated from the Czech.

[35] Tadeusz Kulczycki, Mateusz Kwaśnicki, Jacek Małecki, and Andrzej Stos. Spectral properties of the Cauchy process on half-line and interval. Proc. Lond. Math. Soc. (3), 101(2):589-622, 2010 .

[36] Mateusz Kwaśnicki. Eigenvalues of the fractional Laplace operator in the interval. J. Funct. Anal., 262(5):2379-2402, 2012.

[37] Nick Laskin. Fractional Schrödinger equation. Physical Review E, 66(5):056108, 2002.

[38] Jacques Louis Lions and Enrico Magenes. Non-homogeneous boundary value problems and applications, volume 1. Springer Science \& Business Media, 2012.

[39] Yifei Lou, Xiaoqun Zhang, Stanley Osher, and Andrea Bertozzi. Image recovery via nonlocal operators. Journal of Scientific Computing, 42(2):185-197, 2010.

[40] Yuri Luchko. Fractional Schrödinger equation for a particle moving in a potential well. Journal of Mathematical Physics, 54(1):012111, 2013.

[41] B. M. McCay and M. N. L. Narasimhan. Theory of nonlocal electromagnetic fluids. Arch. Mech. (Arch. Mech. Stos.), 33(3):365-384, 1981.

[42] Ralf Metzler and Joseph Klafter. The restaurant at the end of the random walk: recent developments in the description of anomalous transport by fractional dynamics. J. Phys. A, 37(31):R161-R208, 2004.

[43] P.-A. Raviart and J.-M. Thomas. Introduction à l'analyse numérique des équations aux dérivées partielles. Collection Mathématiques Appliquées pour la Maîtrise. [Collection of Applied Mathematics for the Master's Degree]. Masson, Paris, 1983.

[44] Xavier Ros-Oton and Joaquim Serra. The Dirichlet problem for the fractional Laplacian: Regularity up to the boundary. Journal de Mathématiques Pures et Appliquées, 101(3):275 $-302,2014$.

[45] Xavier Ros-Oton and Joaquim Serra. Local integration by parts and Pohozaev identities for higher order fractional Laplacians. Discrete Contin. Dyn. Syst., 35(5):2131-2150, 2015.

[46] L. Ridgway Scott and Shangyou Zhang. Finite element interpolation of nonsmooth functions satisfying boundary conditions. Math. Comp., 54(190):483-493, 1990.

[47] Raffaella Servadei. The Yamabe equation in a non-local setting. Adv. Nonlinear Anal., 2(3):235-270, 2013.

[48] Raffaella Servadei and Enrico Valdinoci. A brezis-nirenberg result for non-local critical equations in low dimension. Communications on Pure and Applied Analysis, 12(6):2445-2464, 2013.

[49] Raffaella Servadei and Enrico Valdinoci. Variational methods for non-local operators of elliptic type. Discrete Contin. Dyn. Syst., 33(5):2105-2137, 2013.

[50] Raffaella Servadei and Enrico Valdinoci. On the spectrum of two different fractional operators. Proc. Roy. Soc. Edinburgh Sect. A, 144(4):831-855, 2014.

[51] Raffaella Servadei and Enrico Valdinoci. Weak and viscosity solutions of the fractional Laplace equation. Publ. Mat., 58(1):133-154, 2014. 
[52] Raffaella Servadei and Enrico Valdinoci. The Brezis-Nirenberg result for the fractional Laplacian. Trans. Amer. Math. Soc., 367(1):67-102, 2015.

[53] S. A. Silling. Reformulation of elasticity theory for discontinuities and long-range forces. $J$. Mech. Phys. Solids, 48(1):175-209, 2000.

[54] Luis Silvestre. Regularity of the obstacle problem for a fractional power of the Laplace operator. Comm. Pure Appl. Math., 60(1):67-112, 2007.

[55] Enrico Valdinoci. From the long jump random walk to the fractional Laplacian. Bol. Soc. Esp. Mat. Apl. S $\overrightarrow{\mathrm{e}} M A, 49: 33-44,2009$.

[56] Yuan Zhou. Fractional Sobolev extension and imbedding. Transactions of the American Mathematical Society, 367(2):959-979, 2015.

[57] A. Zoia, A. Rosso, and M. Kardar. Fractional Laplacian in bounded domains. Phys. Rev. E (3), 76(2):021116, 11, 2007

J.P. BORTHAGARAY

imAS - CONICET and Departamento de Matemática,

FCEyN - Universidad de Buenos Aires,

Ciudad Universitaria,

Pabellón I (1428) Buenos Aires, Argentina.

E-mail address: jpbortha@dm.uba.ar

Leandro M. Del Pezzo

CONICET AND UTDT

Departamento de Matemáticas y Estadística

Universidad Torcuato Di Tella

Av. Figueroa Alcorta 7350 (C1428BCW)

Buenos Aires, ARGENTINA.

E-mail address: ldelpezzo@utdt.edu

URL: http://cms.dm.uba.ar/Members/ldpezzo/

S. Martínez

imAS - CONiCET and Departamento de Matemática,

FCEyN - Universidad de Buenos Aires,

Ciudad Universitaria,

Pabellón I (1428) Buenos Aires, Argentina.

E-mail address: smartin@dm.uba.ar 